\title{
GRUNSKY-NEHARI INEQUALITIES FOR A SUBCLASS OF BOUNDED UNIVALENT FUNCTIONS
}

\author{
BY \\ D. W. DeTEMPLE( $\left.{ }^{1}\right)$
}

\begin{abstract}
Let $D_{1}$ be the class of regular analytic functions $F(z)$ in the disc $U=\{z:|z|<1\}$ for which $F(0)>0,|F(z)|<1$, and $F(z)+F(\zeta) \neq 0$ for all $z, \zeta \in U$. Inequalities of the Grunsky-Nehari type are obtained for the univalent functions in $D_{1}$, the proof being based on the area method. By subordination it is shown univalency is unnecessary for certain special cases of the inequalities. Employing a correspondence between $D_{1}$ and the class $S_{1}$ of bounded univalent functions, the results can be reinterpreted to apply to this latter class.
\end{abstract}

1. Introduction of the function classes. In this section we provide motivation for the introduction of the function classes which we shall denote in the sequel by $D, D_{1}$, and $D(\beta)$. The univalent functions within these classes (which we indicate by a * superscript) have an interesting connection with the following classes of univalent functions defined on the unit disc $U=\{z:|z|<1\}$.

Class $S$. The functions $g(z)$ which are regular analytic and univalent in $U$, and whose Taylor series expansion about the origin is of the form

$$
g(z)=z+a_{2} z^{2}+\cdots+a_{n} z^{n}+\cdots .
$$

Class $S_{1}$. The functions $f(z)$ which are regular analytic and univalent in $U$, have a Taylor series expansion about the origin of the form

$$
f(z)=b_{1} z+b_{2} z^{2}+\cdots+b_{n} z^{n}+\cdots, \quad b_{1}>0,
$$

and which are bounded (by unity):

$$
|f(z)|<1 \text {. }
$$

Class $S\left(b_{1}\right)$. The functions $f(z)$ in $S_{1}$ which have a prescribed first derivative $f^{\prime}(0)=b_{1}$ at the origin, $0<b_{1} \leqq 1$.

It should be noted that if $f(z) \in S\left(b_{1}\right)$ then $b_{1}^{-1} f(z) \in S$. Under this renormalization $S_{1}$ can be considered to be a dense subset of $S$, and the coefficients $a_{n} \equiv b_{1}^{-1} b_{n}$ belong to the $S$ function $b_{1}^{-1} f(z)=b_{1}^{-1}\left(b_{1} z+\cdots+b_{n} z^{n}+\cdots\right)$. Here dense refers to the topology of uniform convergence on the compact subsets of the disc $U$.

Presented to the Society, June 19, 1971; received by the editors September 9, 1970.

AMS 1969 subject classifications. Primary 3009, 3042, 3043, 3065.

Key words and phrases. Univalent functions, Bieberbach-Eilenberg functions, Grunsky inequalities, Nehari inequalities, subordination.

( $\left.{ }^{1}\right)$ This paper is based upon a doctoral dissertation submitted to Stanford University; the author was supported in part by NSF grant GP-16115 and AF contract AF-0106.

Copyright (C) 1971, American Mathematical Society 
Let $g(z) \in S$ and suppose $u$ is a complex number for which $g(z) \neq 1 / u$. We can then form the function

$$
G(z)=\sqrt{ }(1-u g(z)), \quad G(0)=1,
$$

which is again regular analytic and univalent in $U$. Moreover, the univalency of $g(z)$ assures us that if $G(z)$ takes a value $w$ then it must omit its negative $-w$. This property is characteristic of a function class studied by S. A. Guelfer [3].

Class $D$ (Guelfer). The functions $G(z)$ which are regular analytic in $U$, have a Taylor series expansion about the origin of the form

$$
G(z)=1+\alpha_{1} z+\cdots+\alpha_{n} z^{n}+\cdots,
$$

and which satisfy the property

$$
G(z)+G(\zeta) \neq 0
$$

for all pairs of numbers $z, \zeta \in U$.

Guelfer proved a number of distortion and rotation theorems for $D$ and $D^{*} \equiv\{G(z) \in D: G$ univalent $\}$, and also derived certain estimates for the coefficients $\alpha_{n}$. Inverting the transformation (4) he obtained some of the well-known results for functions $g(z)$ in $S$ which omit a value $1 / u$. For the proofs Guelfer relied on the close relationship which exists between $D$ and the class $C$ of BieberbachEilenberg functions (see [4]).

Class $C$ (Bieberbach-Eilenberg). The functions $E(z)$ which are regular analytic in $U$, and with the properties that $E(0)=0$ and

$$
E(z) E(\zeta) \neq 1
$$

for all pairs $z, \zeta \in U$.

If $G(z) \in D$, we easily see

$$
E(z)=(1-G(z)) /(1+G(z))
$$

is a function in $C$. Thus results known for the class $C$ can be utilized to obtain information about the functions of $D$.

If transformations (4) and (8) are combined, we obtain a correspondence between $S$ and $C^{*}$ which has been considered recently by Hummel and Schiffer [4]. By the area technique they derive for $C^{*}$ functions some general inequalities which are equivalent (via the aforementioned correspondence) to the Garabedian-Schiffer inequalities [2] within the class $S$. The approach through Bieberbach-Eilenberg functions thus provides a proof of elementary character for the GarabedianSchiffer inequalities, in distinction to the original derivation by variational means.

We shall now show how the above considerations can be modified for the case of bounded functions. Let $f(z) \in S_{1}$ and suppose

$$
f(z) \neq \beta^{2} e^{-i \varphi}, \quad 0<\beta<1 \text {. }
$$

The function

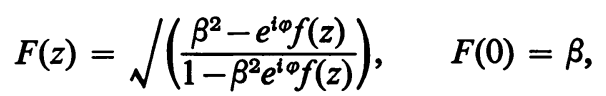


is again regular analytic and univalent in $U$. Moreover, $F(z)$ has the Guelfer property (6) and is bounded: $|F(z)|<1$. In this way we are prompted to define the following two additional function classes.

Class $D_{1}$. The functions $F(z)$ which are regular analytic in $U$ and satisfy the following properties:

$$
\begin{gathered}
F(0)>0, \\
|F(z)|<1, \\
F(z)+F(\zeta) \neq 0
\end{gathered}
$$

for all pairs $z, \zeta \in U$.

Class $D(\beta)$. The functions $F(z)$ in $D_{1}$ which map the origin to a prescribed point $\beta \in(0,1)$, so that

$$
F(0)=\beta \text {. }
$$

Of course $\beta^{-1} F(z) \in D$ whenever $F(z) \in D(\beta)$, and $D_{1}$ is dense in $D$ under this renormalization. More importantly it is to be observed that the transformation (10) may be inverted in a unique way when $F(z)$ is univalent. Indeed, let $F(z) \in D^{*}(\beta)$ and suppose

$$
F(z)=\beta+\beta_{1} z+\cdots+\beta_{n} z^{n}+\cdots .
$$

Then if $\alpha_{1}=\beta^{-1} \beta_{1}=-\left|\alpha_{1}\right| e^{i \varphi}$ we can form

$$
f(z)=e^{i \varphi}\left(\beta^{2}-F^{2}(z)\right) /\left(1-\beta^{2} F^{2}(z)\right) .
$$

The defining properties (12) and (14) assure us $f(z)$ is a bounded regular function which vanishes at the origin. To check univalency, an easy calculation shows that

$$
f(z)-f(\zeta)=-e^{-i \varphi} \frac{(F(z)-F(\zeta))(F(z)+F(\zeta))\left(1-\beta^{4}\right)}{\left(1-\beta^{2} F^{2}(z)\right)\left(1-\beta^{2} F^{2}(\zeta)\right)} .
$$

The univalency of $F(z)$, along with the Guelfer property (13), implies (17) vanishes only if $z=\zeta$. Finally we notice that

$$
f^{\prime}(0)=\left(2 \beta^{2} /\left(1-\beta^{4}\right)\right)\left|\alpha_{1}\right|>0
$$

and so we conclude $f(z) \in S\left(b_{1}\right)$, with $f(z) \neq \beta^{2} e^{-i \varphi}$ and

$$
b_{1}=\left(2 \beta^{2} /\left(1-\beta^{4}\right)\right)\left|\alpha_{1}\right| \text {. }
$$

In the next section we obtain a general area inequality for $D_{1}^{*}$. By means of the correspondence (16) we show how this provides an elementary derivation of inequalities recently obtained by Nehari [5] and DeTemple [1] for the class $S_{1}$. It is to be noted that although Nehari's proof is of the area type to be considered here, his formulation of the inequalities omits the important complementing role of the Guelfer functions $D_{1}^{*}$; in DeTemple on the other hand, the characteristic properties of the $D_{1}^{*}$ class are emphasized, but the variational proof reverts attention again to the class $S_{1}$. 
2. An area theorem for univalent Guelfer functions. For any $F(z) \in D^{*}(\beta)$, we may consider the following power series expansions:

$$
\begin{aligned}
\log \frac{F(z)-F(\zeta)}{z-\zeta} & =\sum_{m, n=0}^{\infty} k_{m n} z^{m} \zeta^{n}, \\
-\log [F(z)+F(\zeta)] & =\sum_{m, n=0}^{\infty} l_{m n} z^{m} \zeta^{n}, \\
\log [1+F(z) \overline{F(\zeta)}] & =\sum_{m, n=0}^{\infty} p_{m n} z^{m} \xi^{n}, \\
-\log [1-F(z) \overline{F(\zeta)}] & =\sum_{m, n=0}^{\infty} q_{m n} z^{m} \xi^{n} .
\end{aligned}
$$

The properties of the class $D^{*}(\beta)$ guarantee the convergence of these series in the bicylinder $U \times U$. It should be observed that the coefficients $k_{m n}$ and $l_{m n}$ are symmetric in their indices, while $p_{m n}$ and $q_{m n}$ are Hermitian symmetric.

Next we define a sequence of rational functions $\Phi_{n}(t)$ by means of the generating function

$$
\log \frac{1-t \beta}{1-t F(\zeta)}=\sum_{n=1}^{\infty} \frac{1}{n} \Phi_{n}(t) \zeta^{n} .
$$

The $\Phi_{n}(t)$ are closely related to the Faber polynomials (see Schiffer [7]). Indeed, if $F_{n}(s)$ is the $n$th Faber polynomial for $[F(z)-\beta]$, then

$$
\Phi_{n}(t)=F_{n}(t /(1-\beta t)) \text {. }
$$

It follows that each $\Phi_{n}(t)$ has a pole only at $t=\beta^{-1}$.

We utilize the functions $\Phi_{n}(t)$ to expand the left sides of (20) in powers of $\zeta$, $|\zeta|$ small, holding $z$ fixed. Comparing equal powers of $\zeta$, we deduce the identities

$$
\begin{aligned}
\sum_{m=0}^{\infty} k_{m 0} z^{m} & =\log \frac{F(z)-\beta}{z}, \\
n \sum_{m=0}^{\infty} k_{m n} z^{m} & =z^{-n}-\Phi_{n}\left[\frac{1}{F(z)}\right] \quad(n \geqq 1), \\
\sum_{m=0}^{\infty} l_{m 0} z^{m} & =-\log [\beta+F(z)], \\
n \sum_{m=0}^{\infty} l_{m n} z^{m} & =\Phi_{n}\left[-\frac{1}{F(z)}\right] \quad(n \geqq 1), \\
\sum_{m=0}^{\infty} p_{m 0} z^{m} & =\log [1+\beta F(z)], \\
n \sum_{m=0}^{\infty} p_{m n} z^{m} & =-\overline{\Phi_{n}[-\overline{F(z)}]} \quad(n \geqq 1), \\
\sum_{m=0}^{\infty} q_{m 0} z^{m} & =-\log [1-\beta F(z)], \\
n \sum_{m=0}^{\infty} q_{m n} z^{m} & =\overline{\Phi_{n}[\overline{F(z)}]}
\end{aligned}
$$


The identities (23) enable us to define a function $q(w)$ for which the inherently positive integral $\iint\left|q^{\prime}(w)\right|^{2} d \tau$ may be evaluated when taken over an appropriate domain. The basic idea is to consider simultaneously both $F(z) \in D^{*}(\beta)$ and its negative

$$
\hat{F}(z)=-F(z)
$$

The ranges of $F(z)$ and $\hat{F}(z)$ are nonoverlapping subdomains of the unit disc $|w|<1$. In particular, by choosing a $\rho(0<\rho<1)$ we may define two nonintersecting simple curves $\gamma$ and $\hat{\gamma}$ inside $|w|<1$ with the parametric representations

$$
w=F\left(\rho e^{i \theta}\right), \quad w=\hat{F}\left(\rho e^{i \theta}\right) .
$$

Along with the unit circumference $|w|=1$ they bound a triply-connected domain $\Delta_{\rho}$ which contains neither $\pm \beta$.

The function $q(w)$ is now defined as follows. Let $x_{0}, y_{0}$ be any two real numbers and let $x_{n}, y_{n}(n=1,2, \ldots, N)$ be any $2 N$ complex numbers. Then let

$$
\begin{aligned}
q(w)= & x_{0} \log \frac{w-\beta}{1-\beta w}-y_{0} \log \frac{w+\beta}{1+\beta w} \\
& -\sum_{m=1}^{N}\left[\frac{x_{m}}{m} \Phi_{m}\left(w^{-1}\right)-\frac{\overline{x_{m}}}{m} \overline{\Phi_{m}(\bar{w})}\right] \\
& +\sum_{m=1}^{N}\left[\frac{y_{m}}{m} \Phi_{m}\left(-w^{-1}\right)-\frac{\overline{y_{m}}}{m} \overline{\Phi_{m}(-\bar{w})}\right] .
\end{aligned}
$$

We observe that $q(w)$ is analytic in $\Delta_{\rho}$ and has there a single-valued real part in view of the realness of $x_{0}$ and $y_{0}$. Using the complex version of Green's identity, we can write

$$
0 \leqq \iint_{\Delta_{\rho}}\left|q^{\prime}(w)\right|^{2} d \tau=\frac{1}{i} \oint_{\partial \Delta_{\rho}} \operatorname{Re}\{q(w)\} d q(w),
$$

where $d \tau$ is the area element in $\Delta_{\rho}$. From (26) we note that $\operatorname{Re}\{q(w)\}=0$ on $|w|=1$. Thus from the parametric representations (25) for the remaining two components $\gamma$ and $\hat{\gamma}$ of the boundary $\partial \Delta_{\rho}$, we see (27) goes over to

$$
I_{1}+I_{2} \leqq 0
$$

where

$$
\begin{aligned}
& I_{1}=\frac{1}{i} \int_{0}^{2 \pi} \operatorname{Re}\left\{q\left[F\left(\rho e^{i \theta}\right)\right]\right\} \frac{d q[F]}{d \theta} d \theta, \\
& I_{2}=\frac{1}{i} \int_{0}^{2 \pi} \operatorname{Re}\left\{q\left[\hat{F}\left(\rho e^{i \theta}\right)\right]\right\} \frac{d q[\hat{F}]}{d \theta} d \theta .
\end{aligned}
$$

The identities (23) enable us to express these integrals in terms of the coefficients of the series (20). Indeed, we find after straightforward calculation that

$$
q[F(z)]=x_{0} \log z-\sum_{n=1}^{N} \frac{x_{n}}{n z^{n}}+\sum_{m=0}^{\infty} A_{m} z^{m},
$$


where

$$
A_{m}=\sum_{n=0}^{N}\left[k_{m n} x_{n}+q_{m n} \bar{x}_{n}+l_{m n} y_{n}+p_{m n} \bar{y}_{n}\right] .
$$

Similarly, we can calculate

$$
q[\hat{F}(z)]=-y_{0} \log z+\sum_{n=1}^{N} \frac{y_{n}}{n z^{n}}-\sum_{m=0}^{\infty} B_{m} z^{m}+\text { imaginary constant, }
$$

where

$$
B_{m}=\sum_{n=0}^{N}\left[k_{m n} y_{n}+q_{m n} \bar{y}_{n}+l_{m n} x_{n}+p_{m n} \bar{x}_{n}\right] .
$$

In view of the developments (30) and (32), the integrals $I_{1}$ and $I_{2}$ may be evaluated to give

$$
\begin{aligned}
I_{1}= & 2 \pi x_{0}^{2} \log \rho-\pi \sum_{n=1}^{N} \frac{1}{n}\left|x_{n}\right|^{2} \rho^{-2 n} \\
& +\pi \sum_{n=1}^{\infty} n\left|A_{n}\right|^{2} \rho^{2 n}+2 \pi x_{0} \operatorname{Re}\left\{A_{0}\right\} \\
I_{2}= & 2 \pi y_{0}^{2} \log \rho-\pi \sum_{n=1}^{N} \frac{1}{n}\left|y_{n}\right|^{2} \rho^{-2 n} \\
& +\pi \sum_{n=1}^{\infty} n\left|B_{n}\right|^{2} \rho^{2 n}+2 \pi y_{0} \operatorname{Re}\left\{B_{0}\right\}
\end{aligned}
$$

From (28) we conclude

$$
\begin{aligned}
2 x_{0} \operatorname{Re}\left\{A_{0}\right\}+2 y_{0} \operatorname{Re}\left\{B_{0}\right\} & +\sum_{n=1}^{\infty} n\left[\left|A_{n}\right|^{2}+\left|B_{n}\right|^{2}\right] \rho^{2 n} \\
& \leqq \sum_{n=1}^{N} \frac{1}{n}\left[\left|x_{n}\right|^{2}+\left|y_{n}\right|^{2}\right] \rho^{-2 n}+2\left(x_{0}^{2}+y_{0}^{2}\right) \log \rho^{-1} .
\end{aligned}
$$

This inequality holds for all $\rho<1$, so we may pass to the limit, $\rho \uparrow 1$. We obtain an area theorem for bounded univalent Guelfer functions

(36) $2 x_{0} \operatorname{Re}\left\{A_{0}\right\}+2 y_{0} \operatorname{Re}\left\{B_{0}\right\}+\sum_{n=1}^{\infty} n\left[\left|A_{n}\right|^{2}+\left|B_{n}\right|^{2}\right] \leqq \sum_{n=1}^{N} \frac{1}{n}\left[\left|x_{n}\right|^{2}+\left|y_{n}\right|^{2}\right]$.

It now becomes useful to introduce a change of variables. Let

$$
\lambda_{n}=\frac{1}{2}\left(x_{n}+y_{n}\right), \quad \mu_{n}=\frac{1}{2}\left(x_{n}-y_{n}\right)
$$

and denote

$$
\begin{array}{ll}
a_{m n}=k_{m n}+l_{m n}, & \alpha_{m n}=k_{m n}-l_{m n}, \\
b_{m n}=q_{m n}+p_{m n}, & \beta_{m n}=q_{m n}-p_{m n} .
\end{array}
$$


Finally we define the new vectors

$$
\begin{aligned}
& C_{m} \equiv \frac{1}{2}\left(A_{m}+B_{m}\right)=\sum_{n=0}^{N}\left[a_{m n} \lambda_{n}+b_{m n} \bar{\lambda}_{n}\right], \\
& D_{m} \equiv \frac{1}{2}\left(A_{m}-B_{m}\right)=\sum_{n=0}^{N}\left[\alpha_{m n} \mu_{n}+\beta_{m n} \bar{\mu}_{n}\right] .
\end{aligned}
$$

Observing that

$$
x_{0} A_{0}+y_{0} B_{0}=2 \lambda_{0} C_{0}+2 \mu_{0} D_{0}
$$

we find from (36) that

(41) $2 \lambda_{0} \operatorname{Re}\left\{C_{0}\right\}+2 \mu_{0} \operatorname{Re}\left\{D_{0}\right\}+\sum_{n=1}^{\infty} n\left[\left|C_{n}\right|^{2}+\left|D_{n}\right|^{2}\right] \leqq \sum_{n=1}^{N} \frac{1}{n}\left[\left|\lambda_{n}\right|^{2}+\left|\mu_{n}\right|^{2}\right]$.

Here we have utilized the parallelogram law for arbitrary complex numbers $a, b$, namely,

$$
|(a+b) / 2|^{2}+|(a-b) / 2|^{2}=\frac{1}{2}\left[|a|^{2}+|b|^{2}\right] .
$$

Having now separated the variables in the inequality, (41) may be split into two independent conditions. For example, taking $\mu_{0}=\mu_{1}=\cdots=\mu_{N}=0$ we find

$$
2 \lambda_{0} \operatorname{Re}\left\{C_{0}\right\}+\sum_{n=1}^{\infty} n\left|C_{n}\right|^{2} \leqq \sum_{n=1}^{N} \frac{\left|\lambda_{n}\right|^{2}}{n} .
$$

Similarly we find

$$
2 \mu_{0} \operatorname{Re}\left\{D_{0}\right\}+\sum_{n=1}^{\infty} n\left|D_{n}\right|^{2} \leqq \sum_{n=1}^{N} \frac{\left|\mu_{n}\right|^{2}}{n} .
$$

We utilize our inequalities as follows. First consider the quantity

$$
\Phi[F]=\operatorname{Re}\left\{\sum_{m, n=0}^{N} a_{m n} \lambda_{m} \lambda_{n}\right\}+\sum_{m, n=0}^{N} b_{m n} \lambda_{m} \bar{\lambda}_{n} .
$$

From (39) we find

$$
\begin{aligned}
\Phi[F] & =\lambda_{0} \operatorname{Re}\left\{C_{0}\right\}+\operatorname{Re}\left\{\sum_{m=1}^{N} \lambda_{m} C_{m}\right\} \\
& \leqq \lambda_{0} \operatorname{Re}\left\{C_{0}\right\}+\left[\left(\sum_{m=1}^{N} \frac{\left|\lambda_{m}\right|^{2}}{m}\right)\left(\sum_{m=1}^{N} m\left|C_{m}\right|^{2}\right)\right]^{1 / 2} \\
& \leqq \lambda_{0} \operatorname{Re}\left\{C_{0}\right\}+\left[\left(\sum_{m=1}^{N} \frac{\left|\lambda_{m}\right|^{2}}{m}\right)^{2}-2 \lambda_{0} \sum_{m=1}^{N} \frac{\left|\lambda_{m}\right|^{2}}{m} \operatorname{Re}\left\{C_{0}\right\}\right]^{1 / 2} \\
& \leqq \lambda_{0} \operatorname{Re}\left\{C_{0}\right\}+\sum_{m=1}^{N} \frac{\left|\lambda_{m}\right|^{2}}{m}-\lambda_{0} \operatorname{Re}\left\{C_{0}\right\} .
\end{aligned}
$$

Here we have used first Schwarz's inequality, then our inequality (42), and finally we have completed the square under the radical. This computation gives us 
THEOREM 1. Let $F(z) \in D_{1}^{*}$ and let $a_{m n}$ and $b_{m n}$ be defined by

$$
\begin{aligned}
\log \frac{F(z)-F(\zeta)}{[F(z)+F(\zeta)](z-\zeta)} & =\sum_{m, n=0}^{\infty} a_{m n} z^{m} \zeta^{n}, \\
\log \frac{1+F(z) \overline{F(\zeta)}}{1-F(z) \overline{F(\zeta)}} & =\sum_{m, n=0}^{\infty} b_{m n} z^{m} \xi^{n} .
\end{aligned}
$$

Then for any real number $\lambda_{0}$ and any $N$ complex numbers $\lambda_{1}, \ldots, \lambda_{N}$,

$$
\operatorname{Re}\left\{\sum_{m, n=0}^{N} a_{m n} \lambda_{m} \lambda_{n}\right\}+\sum_{m, n=0}^{N} b_{m n} \lambda_{m} \bar{\lambda}_{n} \leqq \sum_{m=1}^{N} \frac{\left|\lambda_{m}\right|^{2}}{m} .
$$

Equality is possible only when

$$
\begin{aligned}
\operatorname{Re}\left\{\sum_{n=0}^{N}\left[a_{0 n} \lambda_{n}+b_{0 n} \bar{\lambda}_{n}\right]\right\}=0, & \\
\sum_{n=0}^{N}\left[a_{m n} \lambda_{n}+b_{m n} \bar{\lambda}_{n}\right] & =\frac{\lambda_{m}}{m}, \quad m=1,2, \ldots, N, \\
& =0, \quad m=N+1, N+2, \ldots,
\end{aligned}
$$

and in this case the complement of the union of the domains $F(U)$ and $-F(U)$ with respect to the unit disc has zero area.

Similarly, we find beginning from (43) the

TheOREM 2. Let $F(z) \in D_{1}^{*}$ and let $\alpha_{m n}$ and $\beta_{m n}$ be defined by

$$
\begin{gathered}
\log \frac{F^{2}(z)-F^{2}(\zeta)}{z-\zeta}=\sum_{m, n=0}^{\infty} \alpha_{m n} z^{m} \zeta^{n} \\
-\log \left[1-F^{2}(z) \overline{F^{2}(\zeta)}\right]=\sum_{m, n=0}^{\infty} \beta_{m n} z^{m \bar{\zeta}^{n}} .
\end{gathered}
$$

Then for any real number $\mu_{0}$ and any $N$ complex numbers $\mu_{1}, \ldots, \mu_{N}$,

$$
\operatorname{Re}\left\{\sum_{m, n=0}^{N} \alpha_{m n} \mu_{m} \mu_{n}\right\}+\sum_{m, n=0}^{N} \beta_{m n} \mu_{m} \bar{\mu}_{n} \leqq \sum_{n=1}^{N} \frac{\left|\mu_{n}\right|^{2}}{n} .
$$

Equality is possible only when

$$
\begin{gathered}
\operatorname{Re}\left\{\sum_{n=0}^{N}\left[\alpha_{0 n} \mu_{n}+\beta_{0 n} \bar{\mu}_{n}\right]\right\}=0, \\
\begin{aligned}
\sum_{n=0}^{N}\left[\alpha_{m n} \mu_{n}+\beta_{m n} \bar{\mu}_{n}\right]=\frac{\bar{\mu}_{m}}{m}, \quad m=1, \ldots, N, \\
=0, \quad m=N+1, N+2, \ldots,
\end{aligned}
\end{gathered}
$$

and in this case the complement of the union of the domains $F(U)$ and $-F(U)$, taken with respect to the unit disc, has zero area. 
Each of Theorems 1 and 2 gives analogous results for the unbounded class $D^{*}$ by an obvious limiting procedure. If these inequalities are transformed by (8) into statements on Bieberbach-Eilenberg functions, these results coincide with those obtained by Hummel and Schiffer [4]. For definiteness we state

THEOREM 3. Let $G(z) \in D^{*}$ and let the coefficients $a_{m n}$ be defined by

$$
\log \frac{G(z)-G(\zeta)}{[G(z)+G(\zeta)](z-\zeta)}=\sum_{m, n=0}^{\infty} a_{m n} z^{m} \zeta^{n}
$$

Then for $\lambda_{0}$ real and $\lambda_{1}, \ldots, \lambda_{n}$ any $N$ complex numbers,

$$
\operatorname{Re}\left\{\sum_{m, n=0}^{N} a_{m n} \lambda_{m} \lambda_{n}\right\} \leqq \sum_{m=1}^{N} \frac{\left|\lambda_{m}\right|^{2}}{m} .
$$

To compare with Hummel-Schiffer we need only notice from (8) that

$$
(G(z)-G(\zeta)) /(G(z)+G(\zeta))=(E(z)-E(\zeta)) /(1-E(z) E(\zeta)) .
$$

If in turn $G(z)$ is related to a function $g(z) \in S$ by means of (4), then inequality (57) becomes equivalent to that of Garabedian-Schiffer.

3. Applications. Given a function $f(z) \in S_{1}$ which omits a value $\beta^{2} e^{-i \varphi}$, we have seen that the $F(z)$ given by $(10)$ is in $D^{*}(\beta)$. Hence the inequalities of Theorems 1 and 2 can be restated for these subclasses of $S_{1}$ functions. We find that Theorem 1 becomes equivalent to inequalities of Nehari [5, pp. 325-327] and DeTemple [1], while Theorem 2 is equivalent to the Nehari inequalities as generalized by Schiffer and Tammi [8].

Our inequalities of the preceding section may also be utilized to obtain coefficient and growth estimates for functions $F(z)$ in the class $D(\beta)$. Since the inequalities apply only to univalent functions, the following lemma for the subordination of $D(\beta)$ functions is essential.

Lemma. Let $F(z) \in D(\beta)$. Then there exists for $F(z)$ a univalent majorant $F^{*}(z) \in D^{*}(\beta)$. That is

$$
F(z)=F^{*}[\omega(z)]
$$

where $F^{*} \in D(\beta)$ and $\omega(z)$ is analytic on $U$ with $\omega(0)=0,|\omega(z)|<1$.

The proof, which we omit, can be achieved by the obvious modifications of procedures found in either Rogosinski [6] or Guelfer [3].

A special case of inequality (48) now gives us

THEOREM 4. Let $F(z)=\beta\left(1+\alpha_{1} z+\cdots+\alpha_{n} z^{n}+\cdots\right) \in D(\beta)$. Then

$$
\left|\alpha_{1}\right| \leqq 2\left(1-\beta^{2}\right) /\left(1+\beta^{2}\right) \text {, }
$$


and equality holds only for the functions $w=F(z)$ mapping $U$ onto the half-disc $\{|w|<1, \operatorname{Re} w>0\}$; that is, the functions given by

$$
\frac{F(z)-\beta}{1-\beta F(z)} \frac{1+\beta F(z)}{F(z)+\beta}=\varepsilon z, \quad|\varepsilon|=1 .
$$

Proof. Choose $\lambda_{0}=1$ and $\lambda_{1}=\lambda_{2}=\cdots=\lambda_{n}=0$ in (48). Then for any function $F^{*}(\zeta)=\beta\left(1+\alpha_{1}^{*} \zeta+\cdots\right) \in D^{*}(\beta)$ we have

$$
\operatorname{Re}\left\{a_{00}\right\}+b_{00}=\log \frac{\left|\alpha_{1}^{*}\right|}{2}+\log \frac{1+\beta^{2}}{1-\beta^{2}} \leqq 0,
$$

which is equivalent to (60) in the case of univalent functions. For a general $F(z) \in D(\beta)$ we can write $F(z)=F^{*}(\omega(z))$ by the lemma. But then

$$
\left|\alpha_{1}\right|=\left|\beta^{-1} F^{\prime}(0)\right|=\left|\beta^{-1} F^{* \prime}(0)\right|\left|\omega^{\prime}(0)\right| \leqq\left|\alpha_{1}^{*}\right|
$$

with equality only if $\omega(z)=\varepsilon z,|\varepsilon|=1$.

For equality to hold in (60), we note from (49) and (50) that

$$
C_{0}=a_{00}+b_{00}=\text { imaginary, } \quad C_{m}=a_{m 0}+b_{m 0}=0, \quad m=1,2, \ldots
$$

Subtracting (42) from (40) we then find

$$
q[F(z)]-q[-F(z)]=2 \log z+2 C_{0} .
$$

On the other hand we have from (26) that

$$
q[F(z)]-q[-F(z)]=2 \log \frac{F(z)-\beta}{1-\beta F(z)} \frac{1+\beta F(z)}{F(z)+\beta}+\text { imaginary. }
$$

Comparing (65) and (66) we conclude upon taking real parts that

$$
\log \left|\frac{F(z)-\beta}{1-\beta F(z)} \cdot \frac{1+\beta F(z)}{F(z)+\beta} \cdot \frac{1}{z}\right| \equiv 0 .
$$

From (67) the statements for the extremal functions follow easily.

For a given $f(z) \in S\left(b_{1}\right)$ we can apply Theorem 4 to the function $F(z)$ given by transformation (10). Thus if $f(z) \neq d$ we obtain the well-known inequality

$$
|d| \geqq b_{1}\left(1+\sqrt{ }\left(1-b_{1}\right)\right)^{-2},
$$

with equality holding only for the function given by

$$
f(z)(1+\eta f(z))^{-2}=4|d|(1+|d|)^{-2} z(1+\eta z)^{-2}, \quad \eta=\bar{d} /|d| .
$$

THEOREM 5. Let $F(z) \in D_{1}$. Then

$$
\left|\frac{F^{\prime}(z)}{F(z)}\right| \frac{1+|F(z)|^{2}}{1-|F(z)|^{2}} \leqq \frac{2}{1-|z|^{2}}
$$


holds for all $z \in U$. Equality, at a point $\zeta \in U$, holds only for the functions

$$
F(z)=\frac{\overline{F_{1}(-\zeta)}}{\left|F_{1}(-\zeta)\right|} F_{1}\left(\frac{z-\zeta}{1-\zeta z}\right)
$$

where $F_{1}(z)$ has the form given by (61).

Proof. Let $F(z) \in D_{1}, \zeta \in U$, and form

$$
F_{1}(z)=\frac{\overline{F(\zeta)}}{|F(\zeta)|} F\left(\frac{z+\zeta}{1+\zeta z}\right) .
$$

Then $F_{1}(z) \in D(|F(\zeta)|)$ and

$$
F_{1}^{\prime}(0)=\frac{\overline{F(\zeta)}}{|F(\zeta)|} F^{\prime}(\zeta)\left(1-|\zeta|^{2}\right)
$$

The result now follows by application of Theorem 4 to $F_{1}(z)$.

THEOREM 6. Let $F(z) \in D(\beta)$. Then

$$
\left|\log \frac{F(z)}{\beta} \frac{1-\beta^{2}}{1-F^{2}(z)}\right| \leqq \log \frac{1+|z|}{1-|z|}
$$

and in particular

$$
\begin{gathered}
\frac{1-|z|}{1+|z|} \leqq \frac{\beta}{1-\beta^{2}}\left|\frac{1}{F(z)}-F(z)\right| \leqq \frac{1+|z|}{1-|z|} \\
\left|\arg \left(\frac{1}{F(z)}-F(z)\right)\right| \leqq \log \frac{1+|z|}{1-|z|}
\end{gathered}
$$

Proof. Integrate $(d F / F)\left(\left(1+F^{2}\right) /\left(1-F^{2}\right)\right)$ over the line segment $\langle 0, z\rangle$, and use inequality (70).

For $F(z) \in D(\beta)$ we have seen $G(z)=\beta^{-1} F(z) \in D$. If we replace $F(z)$ by $\beta G(z)$ in our inequalities of this section, then results of Guelfer for the class $D$ are obtained upon sending $\beta \rightarrow 0$.

\section{REFERENCES}

1. D. W. DeTemple, On coefficient inequalities for bounded univalent functions, Ann. Acad. Sci. Fenn. Ser. AI 469 (1970).

2. P. R. Garabedian and M. Schiffer, The local maximum theorem for the coefficients of univalent functions, Arch. Rational Mech. Anal. 26 (1967), 1-32. MR 37 \#1584.

3. S. A. Guelfer, On the class of regular functions which do not take on any pair of values $w$ and -w, Mat. Sb. 19 (61), (1946), 33-46. (Russian) MR 8, 573.

4. J. A. Hummel and M. Schiffer, Coefficient inequalities for Bieberbach-Eilenberg functions, Arch. Rational Mech. Anal. 32 (1969), 87-99. MR 39 \#426.

5. Z. Nehari, Inequalities for the coefficients of univalent functions, Arch. Rational Mech. Anal. 34 (1969), 301-330. MR 40 \#330. 
6. W. Rogosinski, On a theorem of Bieberbach-Eilenberg, J. London Math. Soc. 14 (1939), 4-11.

7. M. Schiffer, Faber polynomials in the theory of univalent functions, Bull. Amer. Math. Soc. 54 (1948), 503-517. MR 10, 26.

8. M. Schiffer and $O$. Tammi, On the coefficient problem for bounded univalent functions, Trans. Amer. Math. Soc. 140 (1969), 461-474. MR 39 \#7088.

\section{Washington State University,}

Pullman, Washington 99163 